\title{
Relação Espacial do Carbono da Vegetação e Matéria Orgânica do Solo na Serra da Mantiqueira
}

\author{
Marcela de Castro Nunes Santos Terra ${ }^{1}$, José Marcio de Mello ${ }^{1}$, \\ Carlos Rogério de Melloº \\ ${ }^{1}$ Departamento de Ciências Florestais, Universidade Federal de Lavras - UFLA, Lavras/MG, Brasil \\ ${ }^{2}$ Departamento de Engenharia, Universidade Federal de Lavras - UFLA, Lavras/MG, Brasil
}

\begin{abstract}
RESUMO
O presente trabalho objetivou analisar o comportamento espacial do estoque de carbono presente no fuste da vegetação (ECV) e da matéria orgânica do solo (MO) em uma floresta ombrófila densa na Serra da Mantiqueira, no Estado de Minas Gerais, por meio de krigagem. Pretendeu-se também testar a utilização da MO superficial como variável auxiliar na predição do ECV com o uso da cokrigagem. Para isso, foram analisados dados georreferenciados de 25 pontos amostrais de $\mathrm{MO}$ em três profundidades, $0-20 \mathrm{~cm}$ (P1), 20-50 cm (P2) e 50-100 cm (P3), e de 12 parcelas amostrais de $400 \mathrm{~m}^{2}$ de vegetação. As variáveis apresentaram estrutura de dependência espacial (maior em ECV e menor em MO em P1) e o semivariograma cruzado refletiu a correlação espacial entre ECV e MO em P1. O mapa de interpolação gerado por cokrigagem foi satisfatório na detecção de tendências da variável ECV, conservando os mesmos padrões do mapa gerado por krigagem ordinária para essa variável. Considera-se que, na área estudada, MO em P1 pode ser utilizada como covariável na caracterização espacial geral do ECV, em situações de subamostragem de ECV.
\end{abstract}

Palavras-chave: mata atlântica, geoestatística univariada, geoestatística multivariada.

\section{Spatial Relationship of Vegetation Carbon Stock and Soil Organic Matter at Serra da Mantiqueira}

\begin{abstract}
We aimed to analyze the spatial behavior of carbon present in the stems of trees (ECV) and soil organic matter $(\mathrm{OM})$ in a dense ombrophilous forest at Serra da Mantiqueira, MG, using kriging. Also we tested the use of superficial organic matter as auxiliary variable in predicting ECV by cokriging. In order to achieve this, we analyzed georeferenced data from 25 sampling points of soil organic matter in three depths, $0-20 \mathrm{~cm}(\mathrm{P} 1), 20-50 \mathrm{~cm}(\mathrm{P} 2)$ and $50-100 \mathrm{~cm}(\mathrm{P} 3)$ and 12 vegetation sample plots of $400 \mathrm{~m}^{2}$. All variables presented spatial dependence structure (higher ECV and lower OM at P1). The cross semivariogram reflected the spatial correlation between ECV and OM at P1. The interpolation map generated by cokriging provided good notion of general ECV trends keeping the same patterns as the one generated by ordinary kriging for this variable. Our findings pointed that in our studied area, $\mathrm{OM}$ at $\mathrm{P} 1$ can be used as a covariate for ECV prediction of where ECV is under sampled.
\end{abstract}

Keywords: atlantic forest, univariate geostatistics, multivariate geostatistics. 


\section{INTRODUÇÃO}

Ecossistemas apresentam variabilidade espacial e temporal em características bióticas e abióticas (Higuchi et al., 2008; Santos et al., 2013; Wojciechowski et al., 2009), e, em se tratando de ecossistemas florestais, é inegável a forte relação entre atributos da vegetação e do solo (Botrel et al., 2002; Baker et al., 2003; Appolinário et al., 2005; Fan et al., 2015).

Os solos funcionam como um sistema de apoio fundamental à vida no ecossistema e desempenham um papel vital na resiliência deste frente às condições geradas pelas mudanças climáticas causadas pelo homem, ou seja, muito mais que um grande reservatório de carbono, o solo funciona como mediador da produtividade e sustentabilidade do ambiente (Brevik, 2009; Rumpel \& Kögel-Knabner, 2010; Brevik, 2013; Parras-Alcántara et al., 2015). Os atributos físico-químicos dos solos são muito influenciados pela matéria orgânica ( $\mathrm{MO})$, a qual corresponde ao maior reservatório de nutrientes para as plantas por estar diretamente relacionada a outras funções no solo, como a fertilidade da qual dependem sociedades e ecossistemas (Mafra et al., 2008; Trumbore \& Czimczik, 2008).

Nesse contexto, o acúmulo de matéria orgânica no solo até níveis capazes de sustentar um fornecimento confiável de nutrientes e água para a biota é fundamental no desenvolvimento dos ecossistemas (Bechtold \& Naiman, 2009), pois garante que, mesmo em solos de baixa fertilidade, florestas exuberantes não apresentem sintomas de deficiência nutricionais, uma vez que o ciclo é praticamente fechado, com a contínua decomposição do material orgânico, associada a uma pequena perda por lixiviação e absorção de elementos do solo (Silva et al., 2007).

Compreender a variabilidade espacial e temporal dos atributos da vegetação, bem como sua relação com variáveis ambientais, é interesse das ciências florestais e áreas afins (Reichstein et al., 2003; Higuchi et al., 2008), uma vez que permite a visualização de cenários que possibilitem prever o comportamento de uma variável diante da abundância ou da ausência de outra, além da possibilidade de estimar determinada variável a partir de outra, cujo custo e/ou tempo de determinação é menor (Landim, 2003).

Diante da necessidade de detectar relações entre as diversas variáveis presentes no ecossistema florestal, a cokrigagem aparece como alternativa metodológica, uma vez que possibilita correlacionar espacialmente variáveis por intermédio do semivariograma cruzado e muitas vezes apresenta ganhos em relação à krigagem ordinária (Angelico, 2006; Wu et al., 2009).

Assim, o presente trabalho objetivou: (1) avaliar o comportamento espacial, via krigagem ordinária, do carbono presente no fuste das árvores e da matéria orgânica do solo em três profundidades em uma microbacia experimental coberta por floresta ombrófila densa na Serra da Mantiqueira, no Estado de Minas Gerais; (2) testar a eficiência da utilização da matéria orgânica da camada superficial do solo (variável de mais fácil obtenção) como covariável na análise espacial, via cokrigagem, do carbono da vegetação, por meio da comparação dos resultados obtidos com os da krigagem ordinária.

\section{MATERIAL E MÉTODOS}

A área de estudo consiste em um remanescente de 13,71 ha de floresta ombrófila densa, localizado em uma microbacia hidrográfica experimental no município de Bocaina de Minas, no Estado de Minas Gerais, entre as coordenadas $22^{\circ} 07^{\prime}$ e $22^{\circ} 09^{\prime}$ de latitude $\mathrm{Se}$ $44^{\circ} 26^{\prime}$ e $44^{\circ} 29^{\prime}$ de longitude W. O local possui clima superúmido A1, segundo classificação de Thornthwaite, o que significa um balanço hídrico positivo ao longo do ano, e é classificado como Cwb, de acordo com Köppen, o que representa ter verões amenos e chuvosos e invernos frios e secos (Sparovek et al., 2007; Carvalho et al., 2008; Pinto et al., 2013). A temperatura média anual é de $16,7^{\circ} \mathrm{C}$, com médias mensais variando de $13,0^{\circ} \mathrm{C}$ em julho a $19,7^{\circ} \mathrm{C}$ em janeiro, e a precipitação média anual é de $2.108 \mathrm{~mm}$, com médias mensais entre $341 \mathrm{~mm}$ no trimestre mais chuvoso (dezembro a fevereiro) a $35 \mathrm{~mm}$ no trimestre mais seco (junho a agosto) (Carvalho et al., 2005). Consiste em uma das mais importantes regiões brasileiras que abrigam nascentes, sendo responsável pela drenagem dos rios Grande e Aiuruoca. O solo dominante na microbacia é o Cambissolo Háplico Distrófico (Menezes et al., 2009), cujo material de origem é o granito-gnaisse, com profundidade classificada como moderadamente profundo $(<1,5 \mathrm{~m})$.

A coleta dos dados foi realizada em junho de 2009. Nessa ocasião, mediu-se em 12 parcelas de 
$400 \mathrm{~m}^{2}(20 \mathrm{~m} \times 20 \mathrm{~m})$ a circunferência a altura do peito (CAP), a 1,30 $\mathrm{m}$ do solo, de todas as árvores com CAP maior ou igual a $15,7 \mathrm{~cm}$ - posteriormente transformados em diâmetro altura do peito (DAP) - e suas alturas totais. As parcelas foram georreferenciadas (latitude, longitude e altitude), utilizando-se um aparelho GPS Magellan, modelo Promark3, trabalhando-se com precisão submétrica $(<1 \mathrm{~m})$. Amostras de solo em três profundidades, $0-20 \mathrm{~cm}$ (P1), 20-50 cm (P2) e 50-100 cm (P3), foram coletadas em 25 pontos georreferenciados da área, dos quais 12 coincidem com as parcelas de amostragem de vegetação (Figura 1).

$\mathrm{O}$ estoque de carbono presente no fuste da vegetação (ECV) foi calculado por meio da equação para a fitofisionomia floresta ombrófila densa, ajustada para as sub-bacias hidrográficas do Rio Grande e do Rio Piracicaba, em Minas Gerais, por Scolforo et al. (2008) (Equação 1).

$\mathrm{C}=\mathrm{e}^{(-11,7511954986+2,1703210582 * \ln (\mathrm{DAP})+0,945801054 * \ln (\mathrm{HT}))}$

em que: C é o estoque de carbono (ton); DAP é o diâmetro a 1,3 $\mathrm{m}$ do solo $(\mathrm{cm})$; HT é a altura total da árvore $(\mathrm{m})$.

Foi realizado o estudo variográfico por meio do semivariograma experimental isotrópico do ECV e da variável matéria orgânica do solo $(\mathrm{MO})$ para as três profundidades. Foram ajustados, pelo método dos mínimos quadrados, para cada situação, os modelos teóricos exponencial, esférico e gaussiano, que representam os modelos mais utilizados na literatura (Cambardella et al., 1994; Mello et al., 2012; Santos et al., 2013; Seidel \& Oliveira, 2013). A seleção de modelos se deu por meio da observação do coeficiente de determinação $\left(\mathrm{R}^{2}\right)$, da soma de quadrados e validação dos resíduos dos modelos e, realizada pelo método da validação cruzada. Os modelos espaciais selecionados permitiram fazer a krigagem ordinária para cada situação analisada.

Feito isso, verificou-se a correlação simples do carbono da vegetação com a MO em P1 e, então, procedeu-se a análise do semivariograma cruzado, sendo a $\mathrm{MO}$ em P1 utilizada como covariável na análise do ECV. Com o semivariograma cruzado é possível verificar a relação entre duas variáveis espacialmente medidas, mostrando se a variabilidade de uma é acompanhada pela variabilidade da outra variável. Se duas variáveis regionalizadas se apresentam correlacionadas espacialmente, então o semivariograma cruzado representa essa correlação e estabelece o vínculo entre elas (Mata et al., 1997; Silva et al., 2003). O estimador para as semivariâncias cruzadas é dado pela Equação 2:

$\hat{\mathrm{Y}} \mathrm{h}_{1,2}=\hat{\mathrm{Y}} \mathrm{h}_{2,1}=\frac{1}{2 \cdot \mathrm{N}(\mathrm{h})}\left\{\sum_{\mathrm{i}-1}^{\mathrm{N}(\mathrm{h})}\left[\mathrm{z}_{1}\left(\mathrm{~s}_{1}\right)-\mathrm{z}_{1}\left(\mathrm{~s}_{1}+\mathrm{h}\right)\right]\left[\mathrm{z}_{2}\left(\mathrm{~s}_{1}\right)-\mathrm{z}_{2}\left(\mathrm{~s}_{1}+\mathrm{h}\right)\right]\right\}$

em que: $z_{1}($.$) representa o valor de uma variável em$ uma dada localização; $z_{2}($.$) é o valor de outra variável$ na mesma posição; h é a distância entre dois pontos amostrados.

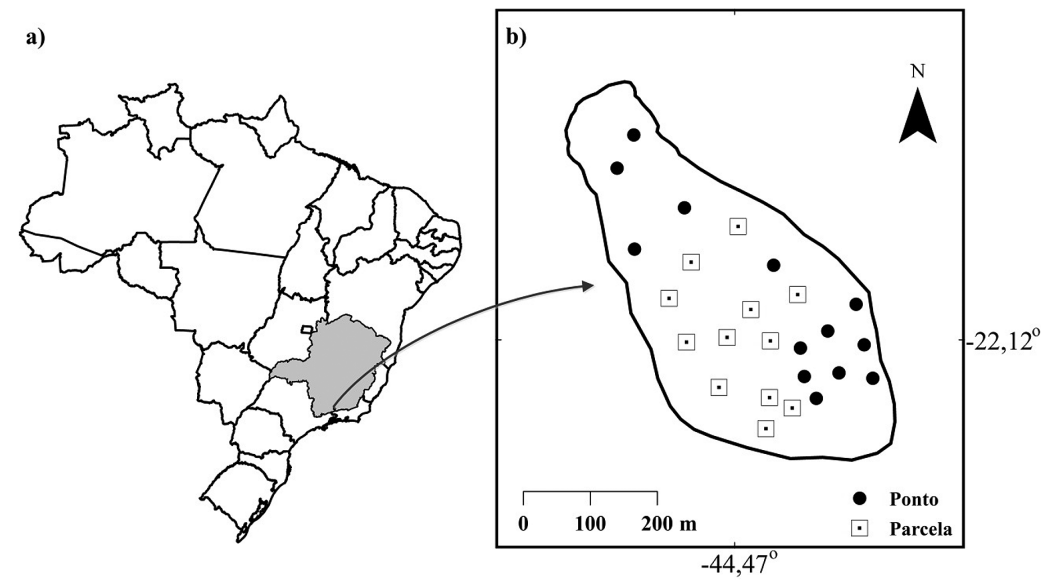

Figura 1. Localização da área de estudos (a) com a posição dos 25 pontos de coleta de amostras do solo e 12 parcelas para coleta de dados da vegetação (b).

Figure 1. Location of the studied area (a) with the position of the 25 soil sample points and 12 vegetation sample plots (b). 
Assim, quando uma só variável z1(.) é analisada, tem-se o semivariograma; quando duas variáveis $\mathrm{z}_{1}\left(\right.$.) e $\mathrm{z}_{2}($.) são analisadas simultaneamente, tem-se o semivariograma cruzado. Feitos os ajustes dos modelos exponencial, esférico e gaussiano ao semivariograma cruzado, foi selecionado o melhor modelo com base nos mesmos critérios do semivariograma simples. Então, com base no modelo espacial selecionado, procedeu-se a cokrigagem, que é uma extensão da técnica de krigagem, utilizada para estimar a variável primária, de difícil mensuração, usando variáveis que são mais facilmente determinadas e correlacionadas com a variável primária (Wu et al., 2009).

A avaliação do desempenho da cokrigagem utilizando MO em P1 como covariável na interpolação do ECV se deu pela validação cruzada. As análises geoestatísticas foram realizadas no software GS+, versão 5.1.1 (Robertson, 1998), e os mapas finais, gerados no ArcGis 9.3 (ESRI, 2006).

\section{RESULTADOS E DISCUSSÃO}

A vegetação apresentou estoque de carbono médio de 39,06 ton.ha ${ }^{-1}$, valor abaixo do esperado para a condição de floresta ombrófila densa. Cunha et al. (2009), estudando biomassa e estoque de carbono e de nutrientes em duas florestas montanas da Mata Atlântica, na região Norte do Estado do Rio de Janeiro, encontraram 65,9 ton.ha ${ }^{-1}$ e 68,4 ton.ha ${ }^{-1}$ de carbono na parte aérea dos fragmentos estudados para árvores com mais de $10 \mathrm{~cm}$ de DAP. No entanto, Ribeiro et al. (2010), quantificando a biomassa e estoque de carbono em uma capoeira da Zona da Mata mineira, obtiveram a estimativa de biomassa das árvores igual a 38,99 ton.ha ${ }^{-1}$ e um estoque de carbono de 19,50 $\pm 8,08$ ton.ha $^{-1}$ para árvores com DAP $>5 \mathrm{~cm}$ e atribuíram os baixos valores encontrados aos distúrbios na área e à presença de gado. Assim, quando comparados aos valores encontrados na literatura, os valores encontrados para estoque de carbono na vegetação no presente estudo apontam para uma condição mais próxima de regeneração do que de floresta madura propriamente. A inferioridade dos valores encontrados deve-se provavelmente aos impactos sofridos na área, como trilhas e presença de gados, que causam variações na densidade e biomassa de indivíduos entre as parcelas amostrais (Piana \& Marsden, 2014; Pereira et al., 2015), e pode estar relacionada também a questões metodológicas envolvidas nas estimativas de carbono (método indireto de determinação).

A variável $M O$ apresentou médias que decresceram da profundidade P1 para P3. Todas as variáveis apresentaram alta variabilidade (Tabela 1).

$\mathrm{O}$ estudo variográfico mostrou que tanto o carbono presente no fuste das árvores quanto a MO, em todas as profundidades, apresentaram estrutura de dependência espacial, com a semivariância aumentando conforme o crescimento da distância entre os pontos amostrados (Figura 2). Em todos os casos o modelo teórico selecionado foi o esférico, em conformidade com Cambardella et al. (1994), que afirmam que propriedades do solo comumente são ajustadas aos modelos esférico e exponencial (Wojciechowski et al., 2009) (Tabela 2).

A contribuição dos modelos espaciais para $\mathrm{MO}$ aumentou conforme o aumento da profundidade, variando de $59 \%$ em P1 até $84 \%$ em P3, o que representa maior dependência espacial na camada mais profunda de solo. Segundo Rumpel \& Kögel-Knabner (2010), em um perfil de solo, a heterogeneidade espacial da distribuição de MO ocorre tanto no sentido horizontal quanto no vertical. Assim, os compostos de $\mathrm{MO}$ não são distribuídos aleatoriamente, mas provavelmente associados à estrutura do solo. Ainda segundo esses autores, três principais processos de incorporação de MO no solo em profundidade foram identificados como sendo responsáveis pela distribuição espacial de compostos orgânicos: (1) fluxo preferencial de carbono

Tabela 1. Valores médios (média) e coeficiente de variação (CV\%) para estoque de carbono da vegetação (ECV) e matéria orgânica do solo (MO) nas três profundidades de solo estudadas (P1, P2 e P3).

Table 1. Mean values (Média) and coefficient of variation (CV\%) for vegetation carbon stock (ECV) and soil organic matter (MO) in the three soil depths considered (P1, P2 and P3).

\begin{tabular}{|c|c|c|c|c|}
\hline & ECV [ton.ha-1] & MO (P1) $\left[\right.$ dag. kg $\left.^{-1}\right]$ & MO (P2) [dag.kg-1] & MO (P3) [dag.kg-1] \\
\hline Média & 39,06 & 6,77 & 4,68 & 3,85 \\
\hline CV (\%) & 83,46 & 42,31 & 36,60 & 43,08 \\
\hline
\end{tabular}



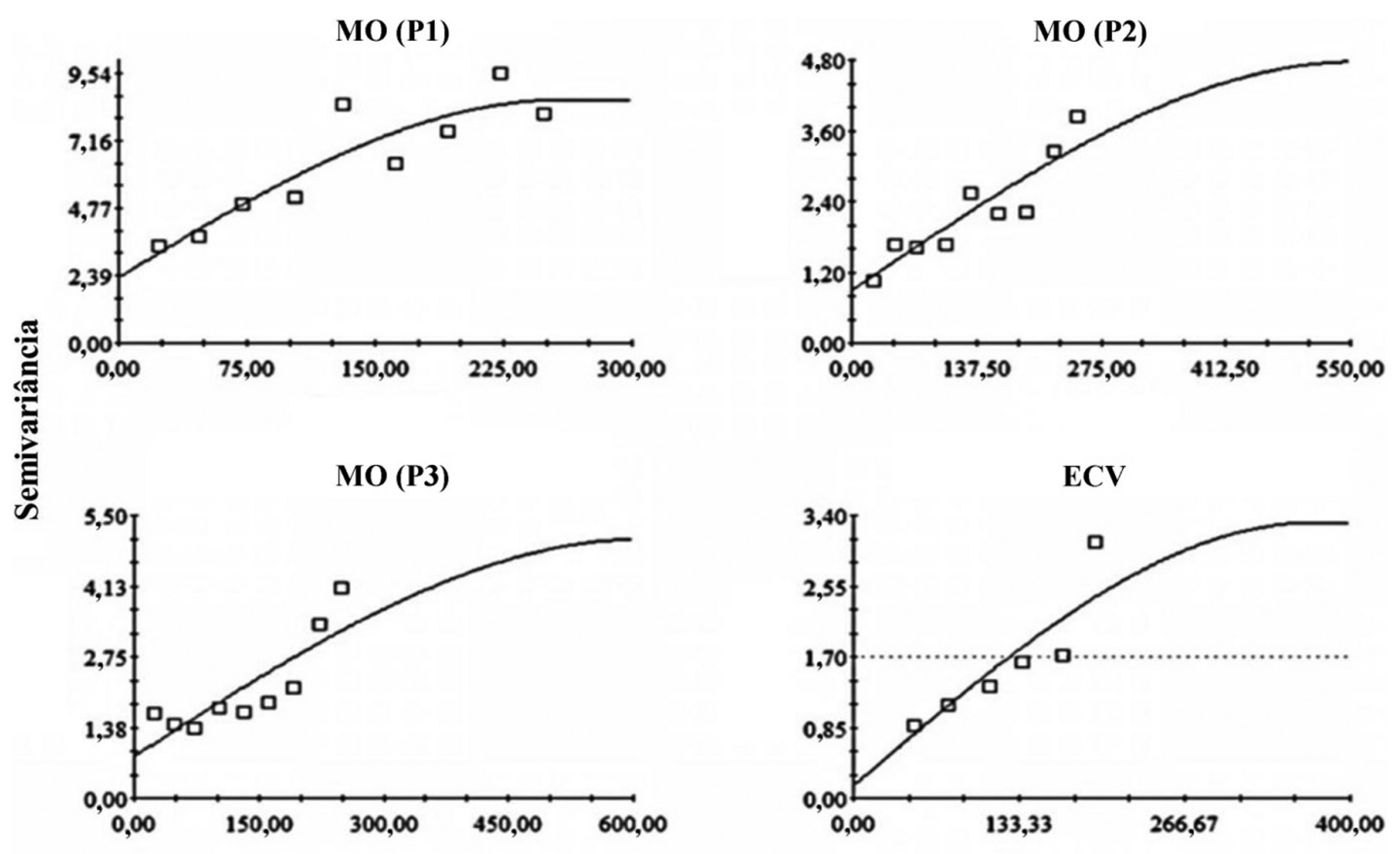

Distância (m)

Figura 2. Semivariogramas isotrópicos para as variáveis estoque de carbono da vegetação (ECV) e matéria orgânica do solo (MO) nas três profundidades de solo estudadas (P1, P2 e P3) e seus modelos espaciais ajustados.

Figure 2. Isotropic semivariograms for the variables vegetation carbon stock (ECV) and soil organic matter (MO) in the three soil depths considered (P1, P2 and P3) and their spatial models fitted.

Tabela 2. Parâmetros dos modelos espaciais ajustados (Co: Efeito Pepita; Co+C: Patamar; Ao: Alcance Prático; $(\mathrm{C} /(\mathrm{Co}+\mathrm{C}))^{\star}$ 100: Contribuição) e medidas de qualidade do ajuste $\left(\mathrm{R}^{2}\right.$ : Coeficiente de Determinação; SQR: Soma de Quadrados do Resíduo) para as variáveis estoque de carbono da vegetação (ECV) e matéria orgânica do solo (MO) nas três profundidades de solo estudadas (P1, P2 e P3).

Table 2. Parameters of spatial models fitted (Co: Nugget Effect; Co + C: Sill; Ao: Practical Range; $\left(\mathrm{C} /(\mathrm{Co}+\mathrm{C})^{\star} 100\right)$ : Contribution) and measures of goodness of fit ( $\mathrm{R}^{2}$ : Coefficient of Determination; SQR: Residual Sum of Squares) for vegetation carbon stock (ECV) and soil organic matter (MO) in the three soil depths considered (P1, P2 and P3).

\begin{tabular}{ccccccccc} 
Variável & Modelo & Co & Co+C & Ao & $(\mathbf{C} / \mathbf{C o}+\mathbf{C})^{\star} \mathbf{1 0 0}$ & $\mathbf{R}^{2}$ & SQR \\
\hline MO (P1) & Esférico & 2,32 & 5,594 & 257,4 & $59 \%$ & 0,82 & 6,78 \\
\hline MO (P2) & Esférico & 0,88 & 4,77 & 555,9 & $82 \%$ & 0,83 & 1,03 \\
\hline MO (P3) & Esférico & 0,81 & 5,038 & 610,9 & $84 \%$ & 0,69 & 2,19 \\
\hline ECV & Esférico & 0,15 & 3,31 & 369,6 & $95 \%$ & 0,77 & 0,709 \\
\hline
\end{tabular}

orgânico dissolvido; (2) comportamento das raízes das plantas; (3) transporte de MO por bioturbação. Sendo assim, o próprio mecanismo que leva a $\mathrm{MO}$ para regiões mais profundas do solo condiciona a estrutura de dependência espacial, além de favorecer o acúmulo desta na porção mais superficial.

Além dos processos de incorporação de $\mathrm{MO}$, sua distribuição é geralmente afetada por outros fatores, tais como o relevo, a textura e o tipo de material de origem do solo do local em questão (Zhang et al.,
2012). Sendo assim, nas camadas mais profundas onde a textura é mais heterogênea e o solo é mais jovem e com pouca $\mathrm{MO}$, há a tendência à distribuição mais agregada da $\mathrm{MO}$, aumentando a dependência espacial do processo. Além disso, as camadas superficiais do solo são menos estáveis e estão sujeitas a intempéries, impactos e fenômenos estocásticos que ocorrem no ambiente florestal, como abertura de clareiras, eventuais incêndios, trilhas, pisoteio de gado, "efeito-borda", dentre outros (Murcia, 1995; Pereira et al., 2015), que 
podem causar alterações pontuais nas características do solo e diminuir o grau de dependência espacial.

Em florestas tropicais, existe estreita relação entre o carbono estocado na vegetação e MO presente nos solos (Mafra et al., 2008; Don et al., 2011; Fan et al., 2015), sendo que a destruição de uma floresta primária ou o processo de impactos que transforma uma floresta primária em secundária causa rápida perda de biomassa, acompanhada por perda de carbono do solo (Don et al., 2011). Tal relação pode ser observada nos mapas de interpolação por krigagem ordinária gerados a partir dos modelos espaciais selecionados para cada variável (Figura 3). As regiões de menor $\mathrm{ECV}$, em geral, coincidem com menores teores de MO, principalmente em P2 e P3, ao passo que as regiões com menor teor de $\mathrm{MO}$ ao sul da bacia coincidem com baixo ECV.

A correlação visual observada nos mapas de krigagem ordinária também é comprovada pela correlação de Pearson entre essas variáveis (Tabela 3) e pelo semivariograma cruzado (Figura 4), o qual indica se a variabilidade de uma variável é acompanhada pela variabilidade de outra. No semivariograma cruzado, a covariável utilizada na estimativa do ECV foi a MO na profundidade de $0-20 \mathrm{~cm}$ (P1). A forma obtida para o gráfico do semivariograma cruzado experimental tem significado diferente do obtido pelo semivariograma simples. Nele, o alcance representa a distância máxima da dependência espacial entre as duas variáveis em estudo, e o patamar, se existir, deve aproximar-se do valor da covariância entre duas variáveis (Silva et al., 2003).

O modelo espacial selecionado para o semivariograma cruzado foi o esférico, uma vez que apresentou melhor
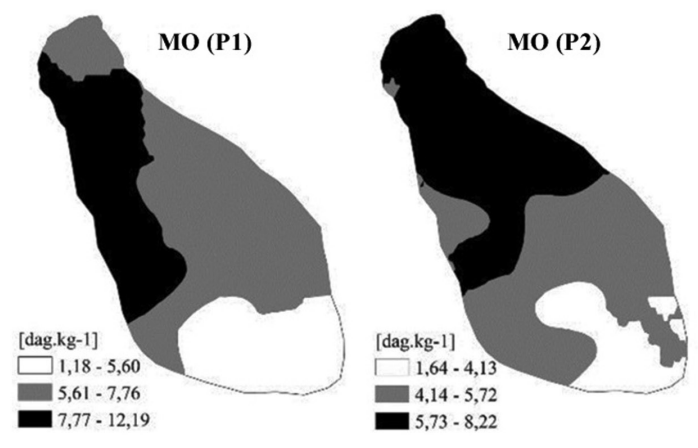

desempenho nos critérios de seleção utilizados. A utilização da covariável MO em P1 para a análise do ECV levou a um alcance alto, mostrando alta distância máxima de dependência espacial entre as variáveis, e a um patamar mais baixo que o do semivariograma de ECV, refletindo a covariância entre as variáveis analisados. As medidas de qualidade de ajuste também refletem a magnitude da correlação existente entre variável primária e covariável (Tabela 4).

A validação cruzada mostra a relação entre valores reais e estimados pelos modelos espaciais por meio de uma equação linear simples (reta). Espera-se que, quanto mais próxima de uma reta que passe pela origem (intercepto igual a 0 ) e com coeficiente angular igual a 1, menores serão os desvios entre as observações reais e as estimadas pela krigagem e cokrigagem. Os semivariogramas apresentaram melhores coeficientes de determinação $\left(\mathrm{R}^{2}\right)$ nas retas de validação cruzada e coeficientes com menores erros-padrão que o semivariograma cruzado (Tabela 5).

Tabela 3. Correlação de Pearson entre a variável matéria orgânica superficial do solo (MO em P1) e as demais variáveis, com destaque para a correlação dessa variável com estoque de carbono da vegetação (ECV).

Table 3. Pearson correlation between the soil superficial organic matter (MO at P1) and the other variables, in particular (highlighted value) the correlation of this variable with the vegetation carbon stock $(\mathrm{ECV})$.

\begin{tabular}{cc} 
& MO (P1) \\
\hline MO (P2) & 0,78 \\
MO (P3) & 0,44 \\
\hline ECV & $\mathbf{0 , 4 2}$ \\
\hline
\end{tabular}
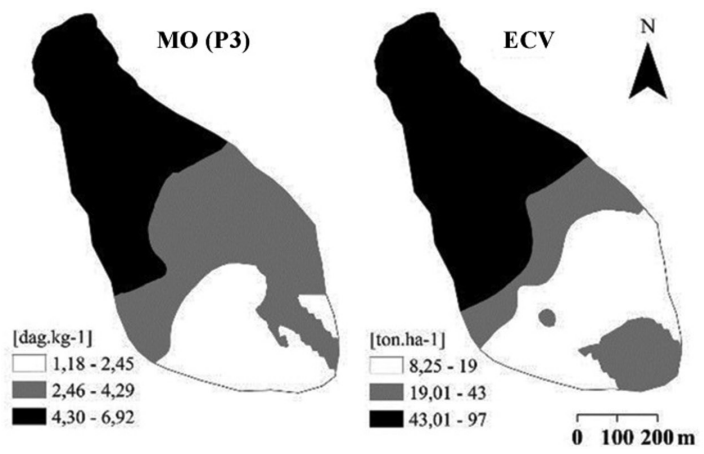

Figura 3. Krigagem ordinária das variáveis estoque de carbono da vegetação (ECV) e matéria orgânica do solo (MO) nas três profundidades de solo estudadas (P1, P2 e P3).

Figure 3. Ordinary kriging of vegetation carbon stock (ECV) and soil organic matter (MO) in the three soil depths considered (P1, P2 and P3). 
Tabela 4. Parâmetros do modelo espacial ajustado ao semivariograma cruzado (Co: Efeito Pepita; Co+C: Patamar; Ao: Alcance Prático; $(\mathrm{C} /(\mathrm{Co}+\mathrm{C}))^{\star} 100$ : Contribuição) e medidas de qualidade do ajuste $\left(\mathrm{R}^{2}\right.$ : Coeficiente de Determinação; SQR: Soma de Quadrados do Resíduo) do estoque de carbono da vegetação (ECV), tendo como covariável matéria orgânica superficial do solo ( $\mathrm{MO}$ em P1).

Table 4. Parameters of the spatial model fitted to cross semivariogram (Co: Nugget Effect; Co + C: Sill; Ao: Practical Range; $(\mathrm{C} /(\mathrm{Co}+\mathrm{C}))^{\star} 100$ : Contribution) and measures of goodness of fit $\left(\mathrm{R}^{2}\right.$ : Coefficient of Determination; SQR: Residual Sum of Squares) of carbon stock of vegetation (ECV) using soil superficial organic matter (MO at P1) as a covariate.

\begin{tabular}{ccccccccc} 
Var. & Modelo & Co & Co+C & Ao & $(\mathbf{C} / \mathbf{C o}+\mathbf{C})^{\star} \mathbf{1 0 0}$ & $\mathbf{R}^{2}$ & SQR \\
\hline ECV & Esférico & 0,58 & 3,16 & 468,8 & $82 \%$ & 0,59 & 0,66 \\
\hline
\end{tabular}

Tabela 5. Estatísticas de precisão oriundas das validações cruzadas produzidas pela krigagem ordinária das variáveis estoque de carbono da vegetação (ECV) e matéria orgânica do solo (MO) nas três profundidades de solo estudadas (P1, P2 e P3) e da cokrigagem de ECV utilizando MO em P1 como covariável.

Table 5. Accuracy statistics derived from cross-validations produced by ordinary kriging of vegetation carbon stock (ECV) and soil organic matter (MO) in the three soil depths considered (P1, P2 and P3) and by cokriging of ECV using ( $\mathrm{MO}$ at $\mathrm{P} 1)$ as a covariate.

\begin{tabular}{lccccc}
\multicolumn{1}{c}{ Variável } & $\begin{array}{c}\text { Coeficiente } \\
\text { Angular }\end{array}$ & Erro-Padrão & $\mathbf{R}^{2}$ & Intercepto & Erro-Padrão \\
\hline MO (P1) & 0,83 & 0,29 & 0,27 & 1,05 & 2,21 \\
MO (P2) & 0,62 & 0,34 & 0,13 & 1,78 & 1,43 \\
MO (P3) & 0,66 & 0,31 & 0,17 & 1,14 & 1,32 \\
ECV & 0,89 & 0,34 & 0,40 & 0,18 & 1,01 \\
ECV (cokriging) & 0,06 & 0,1 & 0,04 & 1,51 & 1,28 \\
\hline
\end{tabular}

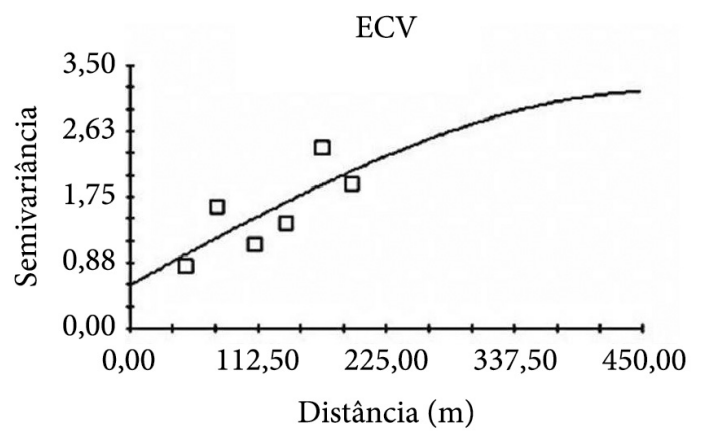

Figura 4. Semivariograma cruzado isotrópico do estoque de carbono da vegetação (ECV) utilizando matéria orgânica superficial ( $\mathrm{MO}$ em $\mathrm{P} 1)$ como covariável.

Figure 4. Isotropic cross semivariogram of carbon stock of vegetation (ECV) using superficial organic matter (MO at P1) as a covariate.

Segundo Viola et al. (2010), para que se obtenham bons resultados com a cokrigagem, é necessário que alterações no comportamento da variável secundária possam explicar, ao menos parcialmente, alterações na variável primária, ou seja, que haja correlação entre elas. No presente estudo, a correlação entre ECV e a covariável utilizada (MO em $\mathrm{P} 1$ ) parece ser fraca para refletir em melhorias significativas na estimativa da variável primária.

É importante lembrar que a quantidade de carbono na vegetação é resultado da interação de muitos outros condicionantes, bióticos e abióticos, além do teor de MO no solo (Ribeiro et al., 2010), o que resulta em grande variabilidade espacial. Essa grande variabilidade pode não ter sido adequadamente captada pelo desenho amostral utilizado neste trabalho, no qual o ECV foi a variável com menor número de unidades amostrais. Algo semelhante ocorre com o teor de $\mathrm{MO}$ no solo, que varia, dentre outros fatores, com o clima, o tipo e idade da vegetação, a textura e regime de saturação hídrica do solo (Rumpel \& Kögel-Knabner, 2010). Portanto, apesar da relação existente entre ECV e MO, deve-se ter em mente que os processos que os caracterizam envolvem muitas outras variáveis e que a utilização de apenas uma dessas variáveis na estimativa da outra pode não ser satisfatória, resultando em muita variação não explicada.

Apesar do baixo desempenho do modelo espacial ajustado ao semivariograma cruzado apontando pela validação cruzada, o mapa de cokrigagem de ECV, gerado com base no modelo espacial ajustado 


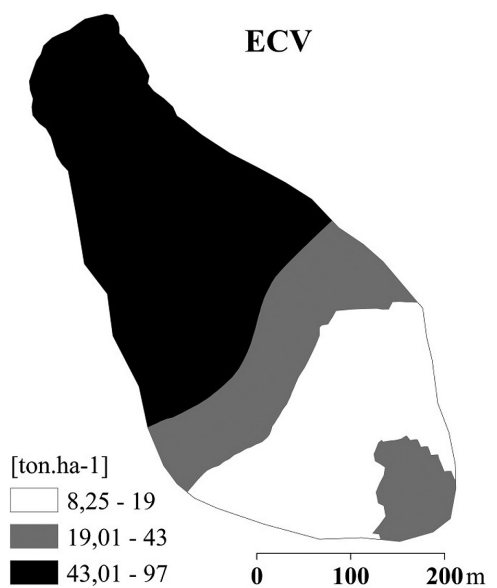

Figura 5. Mapa de cokrigagem do estoque de carbono da vegetação (ECV) utilizando matéria orgânica superficial (MO em P1) como covariável.

Figure 5. Cokriging map of carbon stock of vegetation (ECV) using superficial organic matter (MO at P1) as a covariate.

ao semivariograma cruzado (Figura 5), apresentou padrões semelhantes ao mapa de krigagem ordinária dessa variável, garantindo a sua utilidade, ainda que com baixa capacidade preditiva, como satisfatória ferramenta de detecção de tendências da variável ECV na área.

A amostragem de MO superficial é mais fácil e mais barata, e suas informações podem ser utilizadas como auxiliares na estimativa do ECV, provando ser uma alternativa razoável para quando não há recursos suficientes para coleta de amostras na vegetação. Quando as variáveis são amostradas em espaçamento e intensidade diferentes, pode haver pontos em que apenas a variável auxiliar é medida. Para esses pontos, o valor da variável primária pode ser estimado por meio da variável auxiliar. Com essa vantagem em mente, pode-se desenhar esquemas de amostragem que envolvam ambas as variáveis, em densidades de amostragem bem diferentes, de acordo com o grau de dependência espacial encontrado e com a dificuldade de medição.

\section{CONCLUSÕES}

1. Todas as variáveis se apresentaram estruturadas espacialmente, sendo que o modelo espacial esférico apresentou melhor desempenho na representação da semivariância de todas as variáveis analisadas. A variável ECV foi a que apresentou maior dependência espacial (maior contribuição do modelo espacial ajustado) e a variável MO em P1 apresentou menor dependência espacial. Os mapas de krigagem permitiram visualizar o comportamento espacial das variáveis na área, indicando que áreas com menores teores de $\mathrm{MO}$ no solo em todas as profundidades coincidem de maneira geral com áreas de maior ECV.

2. O ECV se mostrou correlacionado espacialmente com a covariável MO em $\mathrm{P} 1$, sendo essa relação mais bem representada pelo modelo espacial esférico no semivariograma cruzado. Apesar de a validação cruzada apontar baixo desempenho do modelo ajustado, os mapas de krigagem e de cokrigagem de ECV conservaram os mesmos padrões, indicando estimativas satisfatórias do ECV via cokrigagem e, em última instância, que, em situação de subamostragem de ECV, MO em P1 pode ser utilizada com variável auxiliar na detecção de tendências da variável ECV.

3. As complexas relações entre elementos de um ecossistema demandam constantes avanços metodológicos na busca por uma melhor compreensão delas. Esse estudo representa uma nova abordagem para a relação espacial entre dois importantes elementos de um dos mais relevantes e ameaçados ecossistemas brasileiros. Os resultados encontrados têm alta aplicabilidade em estudos que visam à conservação e ao manejo de florestas tropicais.

\section{STATUS DA SUBMISSÃO}

Recebido: 13 set., 2013

Aceito: 8 jun., 2015

\section{AUTOR(ES) PARA CORRESPONDÊNCIA}

\author{
Marcela de Castro Nunes Santos Terra \\ Departamento de Ciências Florestais, \\ Universidade Federal de Lavras - UFLA, \\ Av. Doutor Sylvio Menicucci, 1001, Kennedy, \\ CEP 37200-000, Lavras, MG, Brasil \\ e-mail:marcelacns@gmail.com
}

\section{REFERÊNCIAS}

Angelico JC. Desempenho da co-krigagem na determinação da variabilidade de atributos do solo. Revista Brasileira de Ciencia do Solo 2006; 30(6): 931-936. http://dx.doi. org/10.1590/S0100-06832006000600002. 
Appolinário V, Oliveira AT Fo, Guilherme FAG. Tree population and community dynamics in a Brazilian tropical semideciduous forest. Revista Brasileira de Botanica 2005; 28(2): 347-360. http://dx.doi.org/10.1590/ S0100-84042005000200014.

Baker TR, Burslem DRP, Swaine MD. Associations between tree growth, soil fertility and water availability at local and regional scales in Ghanaian tropical rain forest. Journal of Tropical Ecology 2003; 19(02): 109-125. http://dx.doi. org/10.1017/S0266467403003146.

Bechtold JS, Naiman RJ. A quantitative model of soil organic matter accumulation during floodplain primary succession. Ecosystems 2009; 12(8): 1352-1368. http:// dx.doi.org/10.1007/s10021-009-9294-9.

Botrel R, Oliveira AT Fo, Rodrigues LA, Curi N. Influência do solo e topografia sobre as variações da composição florística e estrutura da comunidade arbóreo-arbustiva de uma floresta estacional semidecidual em Ingaí, MG. Revista Brasileira de Botanica 2002; 25(2): 195-213. http:// dx.doi.org/10.1590/S0100-84042002000200008.

Brevik EC. Soil health and productivity. In: Verheye W, editor. Soils, plant growth and crop production. Oxford: EOLSS: UNESCO; 2009.

Brevik EC. The potential impact of climate change on soil properties and processes and corresponding influence on food security. Agriculture 2013; 3(3): 398-417. http:// dx.doi.org/10.3390/agriculture3030398.

Cambardella CA, Moorman TB, Parkin TB, Karlen DL, Novak JM, Turco RF et al. Field-scale variability of soil properties in central Iowa soils. Soil Science Society of America Journal 1994; 58(5): 1501-1511. http://dx.doi. org/10.2136/sssaj1994.03615995005800050033x.

Carvalho DA, Oliveira AT Fo, van den Berg EVD, Fontes MAL, Vilela EA, Melo Marques JJGS et al. Variações florísticas e estruturais do componente arbóreo de uma floresta ombrófila alto-montana às margens do rio Grande, Bocaina de Minas, MG, Brasil. Acta Botanica Brasílica 2005; 19(1): 91-109. http://dx.doi.org/10.1590/ S0102-33062005000100010.

Carvalho LG, Oliveira MS, Alveas MC, Vianello RL, Sediyama GC, Dantas AAA. Clima. In: Scolforo JR, Carvalho LMT, Oliveira AD, editors. Zoneamento ecológico econômico de Minas Gerais: componentes geofísico e biótico. Lavras: UFLA; 2008.

Cunha GM, Gama-Rodrigues AC, Gama-Rodrigues EF, Velloso ACX. Biomassa e estoque de carbono e nutrientes em florestas montanas da Mata Atlântica na região norte do Estado do Rio de Janeiro. Revista Brasileira de Ciencia do Solo 2009; 33(5): 1175-1185. http://dx.doi.org/10.1590/ S0100-06832009000500011.

Don A, Schumacher J, Freibauer A. Impact of tropical land-use change on soil organic carbon stocks: a metaanalysis. Global Change Biology 2011; 17(4): 1658-1670. http://dx.doi.org/10.1111/j.1365-2486.2010.02336.x.
Environmental Systems Research Institute - ESRI. ArcGIS professional: GIS for desktop. Versão 9.3 [Software]. Redlands; 2006.

Fan H, Wu J, Liu W, Yuan Y, Hu L, Cai Q. Linkages of plant and soil C:N:P stoichiometry and their relationships to forest growth in subtropical plantations. Plant and Soil 2015; 392(1-2): 127-138. http://dx.doi.org/10.1007/ s11104-015-2444-2.

Higuchi P, Oliveira-Filho AT, Bebber DP, Brown ND, Silva AC, Machado ELM. Spatio-temporal patterns of tree community dynamics in a tropical forest fragment in South-east Brazil. Plant Ecology 2008; 199(1): 125-135. http://dx.doi.org/10.1007/s11258-008-9418-x.

Landim PMB. Análise estatística de dados geológicos. 2. ed. São Paulo: UNESP/FEU; 2003.

Mafra AL, Guedes SFF, Klauberg O Fo, Santos JCP, Almeida JA, Rosa JD. Carbono orgânico e atributos químicos do solo em areas florestais. Revista Árvore 2008; 32(2): 217 224. http://dx.doi.org/10.1590/S0100-67622008000200004.

Mata JDV, Gonçalves ACA, Vieira SR, Folegatti MV. Relação entre produtividade e resistência à penetração em área irrigada por pivô central, sob dois sistemas de preparo. Acta Scientiarum 1997; 21(3): 519-525.

Mello CR, Viola MR, Curi N, Silva AM. Distribuição espacial da precipitação e da erosividade da chuva mensal e anual no Estado do Espírito Santo. Revista Brasileira de Ciencia do Solo 2012; 36(6): 1878-1891. http://dx.doi. org/10.1590/S0100-06832012000600022.

Menezes MD, Curi N, Marques JJ, Mello CR, Araújo AR. Levantamento pedológico e sistema de informações geográficas na avaliação do uso das terras em sub-bacia hidrográfica de Minas Gerais. Ciência e Agrotecnologia 2009; 33(6): 1544-1553. http://dx.doi.org/10.1590/S141370542009000600013

Murcia C. Edge effects in fragmented forests: implications for conservation. Trends in Ecology \& Evolution 1995; 10(2): 58-62. http://dx.doi.org/10.1016/S0169-5347(00)88977-6. PMid:21236953.

Parras-Alcántara L, Lozano-García B, Galán-Espejo A. Soil organic carbon along an altitudinal gradient in the Despeñaperros Natural Park, southern Spain. Solid Earth 2015; 6(1): 125-134. http://dx.doi.org/10.5194/ se-6-125-2015.

Pereira JA, Oliveira AT Fo, Eisenlohr PV, Miranda PLS, Lemos JP Fo. Human impacts affect tree community features of 20 forest fragments of a vanishing neotropical hotspot. Environmental Management 2015; 55(2): 296-307. http:// dx.doi.org/10.1007/s00267-014-0387-7. PMid:25344658.

Piana RP, Marsden SJ. Impacts of cattle grazing on forest structure and raptor distribution within a neotropical protected area. Biodiversity and Conservation 2014; 23(3): 559-572. http://dx.doi.org/10.1007/s10531-013-0616-z. 
Pinto LC, Mello CR, Ávila LF. Water quality indicators in the Mantiqueira Range region, Minas Gerais State. Cerne 2013; 19(4): 687-692. http://dx.doi.org/10.1590/ S0104-77602013000400020.

Reichstein M, Rey A, Freibauer A, Tenhunen J, Valentini $\mathrm{R}$, Banza J et al. Modeling temporal and large-scale spatial variability of soil respiration from soil water availability, temperature and vegetation productivity indices. Global Biogeochemical Cycles 2003; 17(4): 1104. http://dx.doi. org/10.1029/2003GB002035.

Ribeiro SC, Jacovine LAG, Soares CPB, Martins SV, Nardelli AMB, Souza AL. Quantificação de biomassa e estimativa de estoque de carbono em uma capoeira da Zona da Mata Mineira. Revista Árvore 2010; 34(3): 495504. http://dx.doi.org/10.1590/S0100-67622010000300013.

Robertson GP. GS+ geoestatistics for the environmental sciences: GS+ user's guide. Plainwell: Gamma Design Software; 1998.

Rumpel C, Kögel-Knabner I. Deep soil organic matter: a key but poorly understood component of terrestrial C cycle. Plant and Soil 2010; 338(1-2): 143-158.

Santos MCN, Mello JM, Mello CR, Ávila LF. Spatial continuity of soil attributes in a Atlantic Forest Remnant in the Mantiqueira Range, MG. Ciência e Agrotecnologia 2013; 37(1): 68-77. http://dx.doi.org/10.1590/S141370542013000100008

Scolforo JRS, Oliveira AD, Acerbi FW Jr. Inventário florestal de Minas Gerais: equações de volume, peso de matéria seca e carbono para diferentes fisionomias da flora nativa. Lavras: UFLA; 2008.

Seidel EJ, Oliveira MS. Proposta de uma generalização para os modelos de semivariogramas exponencial e Gaussiano. Semina: Ciências Exatas e Tecnológicas 2013; 34(1): 125 132. http://dx.doi.org/10.5433/1679-0375.2013v34n1p125.
Silva EAA, Uribe-Opazo MA, Souza EG, Rocha JV. Um estimador robusto e o semivariograma cruzado na análise de variabilidade espacial de atributos de solo e planta. Acta Scientiarum. Agronomy 2003; 25(2): 365-371. http:// dx.doi.org/10.4025/actasciagron.v25i2.1984.

Silva RC, Pereira JM, Araújo QR, Pires AJV, Del Rei AJ. Alterações nas propriedades químicas e físicas de um chernossolo com diferentes coberturas vegetais. Revista Brasileira de Ciencia do Solo 2007; 31(1): 101-107. http:// dx.doi.org/10.1590/S0100-06832007000100011.

Sparovek G, Van Lier QJ, Dourado D No. Computer assisted Koeppen climate classification: a case study for Brazil. International Journal of Climatology 2007; 27(2): 257-266. http://dx.doi.org/10.1002/joc.1384.

Trumbore SE, Czimczik CI. Geology: an uncertain future for soil carbon. Science 2008; 321(5895): 1455-1456. http:// dx.doi.org/10.1126/science.1160232. PMid:18787159.

Viola MR, Mello CR, Pinto DBF, Mello JM, Ávila LF. Métodos de interpolação espacial para o mapeamento da precipitação pluvial. Revista Brasileira de Engenharia Agrícola e Ambiental 2010; 14(9): 970-978. http://dx.doi. org/10.1590/S1415-43662010000900009.

Wojciechowski JC, Schumacher MV, Pires CAF, Madruga PRA, Kilca RV, Brun EJ et al. Geoestatística aplicada ao estudo das características físico-químicas do solo em áreas de floresta estacional decidual. Ciência Florestal 2009; 19(4): 383-391.

Wu C, Wu J, Luo Y, Zhang L, DeGloria SD. Spatial prediction of soil organic matter content using cokriging with remotely sensed data. Soil Science Society of America Journal 2009; 73(4): 1202-1208. http://dx.doi.org/10.2136/ sssaj2008.0045.

Zhang S, Huang Y, Shen C, Ye H, Du Y. Spatial prediction of soil organic matter using terrain indices and categorical variables as auxiliary information. Geoderma 2012; 171-172: 35-43. http://dx.doi.org/10.1016/j.geoderma.2011.07.012. 